\title{
A REGULAR LINDELÖF SEMIMETRIC SPACE WHICH HAS NO COUNTABLE NETWORK
}

\author{
E. S. BERNEY
}

Abstract. A completely regular semimetric space $M$ is constructed which has no $\sigma$-discrete network. The space $M$ constructed has the property that every subset of $M$ of cardinality $2 \boldsymbol{N}_{0}$ contains a limit point of itself; thus, assuming $2 \boldsymbol{\aleph}_{0}=\boldsymbol{N}_{1}, M$ is Lindelöf. It is also shown from the same space $M$ that, assuming $2 \boldsymbol{N}_{0}=\boldsymbol{N}_{1}$, there exists a regular Lindelöf semimetric space $X$ such that $X \times X$ is not normal (hence not Lindelöf).

A. V. Arhangel'skii asks the following question [1, p. 131]: Does every symmetric space have a $\sigma$-discrete network? R. W. Heath asks in [3]: Does every regular Lindelöf semimetric space have a countable network? In this paper Arhangel'skir's question is answered negatively by the construction of a completely regular semimetric (hence symmetric) space $M$ which has no $\sigma$-discrete network. In that same space $M$, every subset of cardinality $2^{\mathbf{s}_{0}}$ contains a limit point of itself, so that, assuming the continuum hypothesis, $M$ would be Lindelöf. Thus, subject to the continuum hypothesis, Heath's question is also answered negatively. Finally it is shown from the same space $M$ that, assuming the continuum hypothesis, there exists a regular Lindelöf semimetric space $X$ such that $X \times X$ is not Lindelöf - in fact not even normal.

A collection of subsets $a$ of a topological space $X$ is said to be a network for $X$ if, given an open set $U$ containing a point $x$, there exists $A \in Q$ such that $x \in A \subseteq U$. A network $Q$ is a $\sigma$-discrete network if $Q$ is the countable union of discrete collections. A topological space $X$ is said to be a semimetric space if there exists a real valued, nonnegative, symmetric function $d$ on $X \times X$ such that:

a. $d(x, y)=0$ iff $x=y$,

b. $x \in \bar{A}$ iff glb $\{d(x, y) \mid y \in A\}=0$.

Let $I$ denote the unit interval. Let $\Delta=\{(x, x) \mid x \in I\}$. If $A$ is a set, let card $A$ denote the cardinality of $A$. Let $\Gamma$ denote the first ordinal such that card $\{\alpha \mid \alpha$ an ordinal, $\alpha<\Gamma\}=2^{\aleph_{0}}$.

THeOREM 1. There is a completely regular semimetric (hence symmetric) space $M$ such that (i) $M$ has no $\sigma$-discrete network, (ii) every

Received by the editors August 29, 1969.

AMS 1968 subject classifications. Primary 5450, 5425.

Key words and phrases. $\sigma$-discrete, network, symmetric space, semimetric space. 
subset of $M$ of cardinality $2^{\boldsymbol{N}_{0}}$ contains a limit point of itself so that, if $\boldsymbol{N}_{1}=2^{\aleph_{0}}, M$ is Lindelöf.

THEOREM 2. If $\boldsymbol{\aleph}_{1}=2^{\aleph_{0}}$, then there is a regular Lindelöf semimetric space $X$ such that $X \times X$ is not normal (hence not Lindelöf).

Lemma. There exists a subset $V$ of $I$ such that:

1. If $U$ is an open subset of $I$, then $\operatorname{card}(U \cap V)=2 \aleph_{0}$.

2. There exists no pair $f$ and $D$ such that:

(i) $D \subseteq V$ and card $D=2^{\mathrm{s}_{0}}$,

(ii) $f: D \rightarrow V$,

(iii) either $f$ is strictly increasing and $\{(x, f(x)) \mid x \in D\}$ is bounded away from $\Delta$, or $f$ is strictly decreasing.

Proof of Lemma. Let $e$ denote the set of all closed subsets of $I$. If $A \in \mathfrak{C}$, let $\mathfrak{F}(A)$ denote the set of all monotone functions from $A$ into $I$ such that point-inverses are at most countable. Let

$$
\mathcal{F}=\bigcup\{F(A) \mid A \in \mathcal{C}\} .
$$

It is easily shown that $\operatorname{card}(\mathcal{F})=2^{\boldsymbol{N}_{0}}$. Let $\mathcal{U}$ denote the set of all open subsets of $I$. Let $\mathcal{U}=\left\{\mathcal{U}_{\alpha}: \alpha<\Gamma\right\}$ and $\mathcal{F}=\left\{f_{\alpha}: \alpha<\Gamma\right\}$ be wellorderings of $\mathcal{U}$ and $\mathcal{F}$, recalling that $\Gamma$ is the first ordinal of cardinal $2^{\aleph_{0}}$. Define a set $V$ as follows. Select a point $x_{1}$ from $U_{1}$. For each $\beta, 1<\beta<\Gamma$, such that $x_{\alpha}$ is defined for all $\alpha<\beta$, pick $x_{\beta}$ to be some point of $U_{\beta}$ such that

$$
x_{\beta} \notin\left[\cup\left\{f_{\alpha}^{-1}\left(x_{\tau}\right) \mid \alpha \leqq \beta, \tau<\beta\right\} \cup\left\{x_{\tau} \mid \tau<\beta\right\} \cup\left\{f_{\alpha}\left(x_{\tau}\right) \mid \alpha \leqq \beta, \tau<\beta\right\}\right] .
$$

Let $V=\left\{x_{\alpha} \mid \alpha<\Gamma\right\}$. The proof that $V$ satisfies condition 1 is obvious. To see that condition 2 is satisfied, suppose not; then there exists a pair $f$ and $D$ satisfying 2(i), 2(ii), and 2(iii). Assume $f$ is strictly decreasing (the argument is similar if $f$ is strictly increasing and $\{(x, f(x)) \mid x \in D\}$ is bounded away from $\Delta$ ). Extend $f$ to $\bar{D}$ (call the extension $\hat{f}$ ) as follows: if $t \in \bar{D}$, then

$$
\begin{array}{rlrl}
\hat{f}(t) & =f(t) & & \text { if } t \in D, \\
& =\operatorname{lub}_{x>t ; x \in D} f(x) & \text { if } t \text { is a limit point of } D \text { from above and } t \notin D, \\
& =\operatorname{glb}_{x<t ; x \in D} f(x) & \text { if } t \text { is not a limit point of } D \text { from above and } t \notin D .
\end{array}
$$

It is easily checked that $\hat{f} \in \mathcal{F}$. Therefore, for some ordinal $\alpha, \hat{f}=f_{\alpha}$. Now $f_{\alpha}$ has the property that there exists at most one $x_{\theta}$ in $D$ such that $f_{\alpha}\left(x_{\theta}\right)=x_{\theta}$ (in the case of $f_{\alpha}$ strictly increasing etc., $\{(x, f(x)) \mid x \in D\}$ being bounded away from $\Delta$ guarantees for every $\left.\theta, \theta<\Gamma, f_{\alpha}\left(x_{\theta}\right) \neq x_{\theta}\right)$. 
Now there exist $x_{\gamma}, x_{\beta} \in D$ such that $\gamma, \beta>\max \{\theta, \alpha\}, \gamma \neq \beta$, and $f_{\alpha}\left(x_{\gamma}\right)=x_{\beta}$. For if not, then $f_{\alpha}$ is a one-to-one correspondence between $\left\{x_{\tau} \mid \tau>\max (\theta, \alpha)\right\}$ and $\left\{x_{\tau} \mid \tau \leqq \max (\theta, \alpha)\right\}$, which is a contradiction since $\operatorname{card}\left[\left\{x_{\tau} \mid \tau>\max (\theta, \alpha)\right\}\right]>\operatorname{card}\left[\left\{x_{\tau} \mid \tau \leqq \max (\theta, \alpha)\right\}\right]$. Thus, let $x_{\gamma}, x_{\beta}$ be two such points. If $\gamma<\beta$, then we have a contradiction to the definition of $V$. If $\gamma>\beta$, as $x_{\gamma} \in f_{\alpha}^{-1}\left(x_{\beta}\right)$, we also have a contradiction to the definition of $V$. Thus, the proof is complete.

Proof of Theorem 1. Let $V$ be as in the Lemma. Choose $M$ to be a subset of $V \times V-\Delta$ such that: (1) if $U$ is open in $I \times I$, then $\operatorname{card}(U \cap M)$ is $2^{\aleph_{0}}$, and (2) if $\Pi_{1}$ and $\Pi_{2}$ denote the two projection functions on $V \times V$ into $V$, then for each $m \in M, \Pi_{i}(m) \notin$ $\Pi_{i}(M-\{m\}), i=1,2$. Let $d$ denote the usual Euclidean metric of $I \times I$. Define $d^{*}$ on $M \times M$ as follows: if $(a, b),(c, d) \in M$, then

$$
\begin{aligned}
d^{*}[(a, b),(c, d)] & =d[(a, b),(c, d)] & & \text { if } a \leqq c \text { and } b \leqq d \text { or } a \geqq c \text { and } b \geqq d, \\
& =1 & & \text { if } a<c \text { and } b>d \text { or } a>c \text { and } b<d .
\end{aligned}
$$

It is easily shown that $d^{*}$ generates a completely regular semimetric topology on $M$ (denoted by $M\left(d^{*}\right)$ ) with semimetric $d^{*}$ by defining: $A$ is closed in $M$ iff $A \supseteq\left\{x \in M \mid d^{*}(x, A)=0\right\}$. We now show that $M$ (with the $M\left(d^{*}\right)$ topology) has the property that every subset $A$ of cardinality $2^{N_{0}}$ has a limit point in $A$. Let $A$ be a subset of $M$ such that card $A$ is $2^{N_{0}}$. Suppose $A$ is discrete in itself. Then there exists a subset $A^{\prime}$ of $A$ such that card $A^{\prime}=2^{\mathrm{N}_{0}}$ and a positive number $\delta$ such that $(c, d),(a, b) \in A^{\prime}$ implies $d^{*}[(a, b),(c, d)]>\delta$. Let $(p, q) \in A^{\prime}$ such that for each Euclidean disk $S$ about $(p, q), \operatorname{card}\left(S \cap A^{\prime}\right)=2^{\mathrm{N}_{0}}$. Let $S$ be a Euclidean disk of radius less than $\delta / 2$ about $(p, q)$. If $(a, b),(c, d) \in S \cap A^{\prime}$ and $a<c$, then $b>d$. For if not, then $a<c$ and $b<d$ implies $d^{*}[(a, b),(c, d)]=d[(a, b),(c, d)]<\delta$, a contradiction. Note $S \cap A^{\prime}$ is a strictly decreasing function on $\Pi_{1}\left(S \cap A^{\prime}\right)$ into $V$ and $\operatorname{card}\left[\Pi_{1}\left(S \cap A^{\prime}\right)\right]=2^{N_{0}}$, a contradiction to the definition of $V$. Hence part (ii) of Theorem 1 is true. We now show that $M$ has no $\sigma$-discrete network. Suppose it does, say $Q$ where $Q=U\left\{B_{i} \mid i=1,2, \cdots\right\}$. By part (ii) of Theorem 1, card $B_{i}<2^{\aleph_{0}}$, hence card $Q<2^{\text {No}_{0}}$. Let $N(x, y)$ denote the $d^{*}$ sphere of radius $\frac{1}{2}$ about $(x, y)$. There exist a subset $M^{1}$ of $M$ and an $A$ in $Q$ such that $\operatorname{card}\left(M^{1}\right)=2^{\aleph_{0}}$ and $(a, b) \in M^{1} \mathrm{im}$ plies $(a, b) \in A \subseteq N(a, b)$. Let $(p, q) \in M^{1}$ such that, if $S$ is a Euclidean disk about $(p, q)$, then $\operatorname{card}\left(S \cap M^{1}\right)=2^{\aleph_{0}}$. Let $S$ be a Euclidean disk of radius $r$ about $(p, q)$ such that $0<r<\min \left\{\frac{1}{4}, \frac{1}{2} d[(p, q), \Delta]\right\}$. Let $(a, b),(c, d) \in S \cap M^{1}$. If $a<c$, then $b<d$. For if not, there exists points $(a, b)$ and $(c, d)$ in $S \cap M^{1}$ such that $a<c$ and $b>d$. Then $(c, d) \notin N(a, b)$, but $(c, d) \in A \subseteq N(a, b)$ which is a contradiction. Thus 
$S \cap M^{1}$ is a strictly increasing function on $\Pi_{1}\left(S \cap M^{1}\right)$ into $V$ such that $d\left[S \cap M^{1}, \Delta\right]>0$ and card $\Pi_{1}\left(S \cap M^{1}\right)=2^{\aleph_{0}}$, which is a contradiction to the definition of $V$. Hence $M$ (with the $M\left(d^{*}\right)$ topology) has no $\sigma$-discrete network.

Proof of Theorem 2. Define $d^{\prime}$ on $M \times M$ by

$$
\begin{aligned}
d^{\prime}[(a, b),(c, d)] & =d[(a, b),(c, d)] & & \text { if } a \leqq c \text { and } b \geqq d \text { or } a \geqq c \text { and } b \leqq d, \\
& =1 & & \text { if } a<c \text { and } b>d \text { or } a>c \text { and } b<d .
\end{aligned}
$$

Then, again, we have a regular Lindelöf semimetric space (with semimetric $d^{\prime}$ ) which has no countable network (similar arguments as in the proof of Theorem 1). Denote the $d^{\prime}$ semimetric topology by $M\left(d^{\prime}\right)$. Let $X_{1}$ (respectively $X_{2}$ ) denote $M$ with the $M\left(d^{*}\right)$ (respectively $M\left(d^{\prime}\right)$ ) topology. Let $X$ be the free union [2] of $X_{1}$ and $X_{2}$ (i.e., $X=\{(i, z): z \in M, i=1,2\}$ with the topology generated by $\left\{\{i\} \times U: U\right.$ is open in $\left.\left.X_{i}, i=1,2\right\}\right)$. Then $X$ is a regular Lindelöf semimetric space which has no countable network and $X \times X$ is not normal. To see $X \times X$ is not normal (and hence not Lindelöf), consider the set $R=\{((1, z),(2, z)): z \in M\}$ which is a subset of $X \times X$. It is easily shown that $R$ is closed in $X \times X$. We now show that $R$ is discrete in itself. Let $z \in M$. Let $((1, z),(2, z)) \in R$. Let $S_{1}$ denote the $d^{*}$ semimetric sphere of radius $\frac{1}{2}$ about $z, S_{2}$ denote the $d^{\prime}$ semimetric sphere of radius $\frac{1}{2}$ about $z, S_{1}^{1}=\{1\} \times S_{1}$, and $S_{2}^{1}=\{2\} \times S_{2}$. Let $z$ $=(x, y)$. Suppose $((1, c),(2, c)) \in R \cap\left(S_{1}^{\prime} \times S_{2}^{\prime}\right)$. If $c=(a, b)$, then $a$ $\leqq x$ and $b \leqq y$ or $a \geqq x$ and $b \geqq y$ as $(1, c) \in S_{1}^{\prime}$. Similarly $a \leqq x$ and $y \leqq b$ or $a \geqq x$ and $y \geqq b$ as $(1, c) \in S_{2}^{\prime}$. Hence $a=x$ and $b=y$. Therefore $R \cap\left(S_{1}^{\prime} \times S_{2}^{\prime}\right)=\{((1, z),(2, z))\}$ and $R$ is discrete in itself. Note $X \times X$ is separable. Thus, by Theorem 1 of [4], $X \times X$ is not normal.

\section{REFERENCES}

1. A. V. Arhangel'skiY, Mappings and spaces, Uspehi Mat. Nauk 21 (1966), no. 4 (130), 133-184 = Russian Math. Surveys 21 (1966), no. 4, 115-162. MR 37 \#3534.

2. J. Dugundji, Topology, Allyn and Bacon, Boston, Mass., 1966. MR 33 \#1824.

3. R. W. Heath, On certain first-countable spaces, Topology Seminar (Wisconsin, 1965), Ann. of Math. Studies, no. 60, Princeton Univ. Press, Princeton, N.J., 1966, pp. 103-113.

4. F. B. Jones, Concerning normal and completely normal spaces, Bull. Amer. Math. Soc. 43 (1937), 671-679.

Arizona State University, Tempe, Arizona 85281 